\title{
Commentary on 'Microbiome diversity predicts surgical success in patients with rectovaginal fistula'
}

\author{
Abdelmageed Abdelrahman ${ }^{1}$ \\ Received: 2 November 2020 / Accepted: 16 November 2020 / Published online: 27 November 2020 \\ (C) The International Urogynecological Association 2020
}

This is a single-institution pilot study with the primary aim of characterizing the vaginal and rectal microbiome in patients with rectovaginal fistula planning to undergo surgical treatment between 1 January 2018-31 May 2019. Secondary aims included to collectively and longitudinally evaluate changes in the microbiome and in quality of life measurements through the perioperative course. Exclusion criteria included patients with colovaginal and enterovaginal fistula. Additional exclusion criteria included: current pregnancy, current or recent history (within 4 weeks) of antibiotic use, history of chemotherapy within 2 years of fistula surgery, prior pelvic or abdominal radiotherapy, and current or planned intestinal diversion. Rectal and vaginal samples were obtained from 14 patients with rectovaginal fistula preoperatively, immediately following surgery, $6-8$ weeks postoperatively and at the time of fistula recurrence.

There was a total of eight fistula recurrences following surgery. The rectal and vaginal microbiome in patients undergoing successful fistula repair was different from those with recurrence. This was also characterized by a higher species diversity. Thirty-one taxa were enriched in patients undergoing successful repair, including Bacteroidetes; Alistipes and
Rikenellaceae as well as Firmicutes; Subdoligranulum, Ruminococcaceae UCG-010 and NK4A214 group. The authors concluded that the association of higher vaginal diversity with a favourable outcome has not been previously described.

This is an interesting study where the dominant taxa of the rectal and vaginal microbiome have been characterized for the first time in patients with rectovaginal fistula. Limitations of this pilot study include the small sample size. Negative associations elicited from the study should also be interpreted with caution as this may result from under-powering. Another limitation is the relatively homogeneous ethnic distribution of patients included in the study.

\section{Compliance with ethical standards}

Conflicts of interest None.

Publisher's note Springer Nature remains neutral with regard to jurisdictional claims in published maps and institutional affiliations.
Abdelmageed Abdelrahman

abdelmageed@hotmail.co.uk

1 Department of Urogynaecology, Liverpool Women's Hospital NHS Foundation Trust, Liverpool, UK 